population? Pediatrics April 2006;117:601-609). (Respond: Helen Schneider PhD, Department of Economics, University of Texas at Austin, BRB 3.116, Austin, TX 78712).

COMMENT. The likelihood of ADHD diagnosis is dependent on a child's gender and race, the presence of a biologic father in the home, the teacher's color and age, the season and geographic region of birth, the socio-economic status, and school standards and performance accountability.

\title{
EFFECT OF LONG-ACTING STIMULANTS ON GROWTH
}

The effect of OROS methylphenidate (OROS MPH, Concerta) [34-43 mg daily; 1.1$1.2 \mathrm{mg} / \mathrm{kg} /$ day] on the height and weight of 178 children, ages 6 to 13 years, treated for at least 21 months for attention-deficit/hyperactivity disorder (ADHD) was evaluated at the Massachusetts General Hospital, Boston. Subjects ( $86 \%$ having received stimulant therapy previously) were at the expected height for their age at baseline and somewhat heavier than expected. Height and weight were measured monthly during the first year of treatment and then every 3 months. Height steadily increased throughout the study, but subjects were on average $0.23 \mathrm{~cm}$ less than expected at month 21 . Weight showed no increase and body mass index (BMI) decreased slightly in the first 4 months of treatment; weight and BMI remained constant after 4 months, and were on average $1.23 \mathrm{~kg}$ less than expected at 21 months. Those taking drug holidays ( $29 \%$ omitting treatment for $>30$ days vs $71 \%$ for $<30$ days during the study) showed trends toward lesser decreases in weight and growth. Among 167 subjects who discontinued treatment early, $36(8.8 \%)$ reported lack of effectiveness, $31(7.6 \%)$ adverse events, $29(7.1 \%)$ lost to follow-up, and $28(6.9 \%)$ failed to follow protocol. Growth measurements were not obtained at both baseline and 21 months in 51. (Spencer TJ, Faraone $\mathrm{SV}$, Biederman J, et al. Does prolonged therapy with a long-acting stimulant suppress growth in children with ADHD? J Am Acad Child Adolesc Psychiatry May 2006;45:527-537). (Respond: Dr Thomas J Spencer, Massachusetts General Hospital, 15 Parkman Street, WACC 725, Boston, MA 02114).

COMMENT. Although the authors conclude that the effects of prolonged OROS MPH on growth of ADHD children are clinically insignificant, and significant decreases in weight are limited to the first months of therapy, their data show some small adverse effects in the group as a whole. The results of this study should not negate the importance of monitoring children carefully for more significant potential effects on growth in the individual child. Furthermore, the possible benefits of drug holidays in lessening long-term adverse effects should not be dismissed by the results of this study; a drug holiday of only $5 \%$ of the time, as used by the authors, is considerably shorter than the 130 day (35\%) annual drug holiday (on weekends and vacations), frequently used in conservative regimens.

Effects of MPH vs mixed amphetamine salts (Adderall) on height in children with ADHD treated for 1 to 3 years were similar; linear regression found a small but statistically significant negative effect on height. Both drugs caused a significant decrease in weight and BMI, patients becoming thinner over time, and this effect was more pronounced with mixed amphetamine salts (Pliszka SR, et al. J Am Acad Child Adolesc Psychiatry May 2006;45:520-526). 